\title{
Spectroscopy of globular clusters in the low-luminosity spiral galaxy NGC $45^{\star}$
}

\author{
M. D. Mora ${ }^{1,3}$, S. S. Larsen ${ }^{2}$, and M. Kissler-Patig ${ }^{3}$ \\ 1 Departamento de Física y Astronomía, Facultad de Ciencias, Universidad de Valparaíso. Av. Gran Bretaña 1111, Valparaíso, Chile \\ e-mail: mmora@dfa.uv.cl \\ 2 Astronomical Institute, University of Utrecht, Princetonplein 5, 3584 CC, Utrecht, The Netherlands \\ e-mail: S.S.Larsen@uu.nl \\ 3 European Southern Observatory, Karl-Schwarzschild-Strasse 2, 85748 Garching bei München, Germany \\ e-mail: mkissler@eso.org
}

Received 28 April 2008 / Accepted 24 July 2008

\begin{abstract}
Context. Extragalactic globular clusters have been studied in elliptical galaxies and in a few luminous spiral galaxies, but little is known about globular clusters in low-luminosity spirals.

Aims. Past observations with the ACS have shown that NGC 45 hosts a large population of globular clusters (19), as well as several young star clusters. In this work we aim to confirm the bona fide globular cluster status for 8 of 19 globular cluster candidates and to derive metallicities, ages, and velocities.

Methods. VLT/FORS2 multislit spectroscopy in combination with the Lick/IDS system was used to derive velocities and to constrain metallicities and $[\alpha / \mathrm{Fe}]$ element ratio of the globular clusters.

Results. We confirm the 8 globular clusters as bona fide globular clusters. Their velocities indicate halo or bulge-like kinematics, with little or no overall rotation. From absorption indices such as $\mathrm{H} \beta, \mathrm{H} \gamma$, and $\mathrm{H} \delta$ and the combined $[\mathrm{MgFe}]^{\prime}$ index, we found that the globular clusters are metal-poor $[\mathrm{Z} / \mathrm{H}] \leq-0.33$ dex and $[\alpha / \mathrm{Fe}] \leq 0.0$ element ratio. These results argue in favor of a population of globular clusters formed during the assembling of the galaxy.
\end{abstract}

Key words. galaxies: individual: NGC 45 - galaxies: star clusters

\section{Introduction}

Globular clusters are present in almost all kinds of galaxies. Observations of extragalactic globular cluster systems have shown that globular cluster systems can often be divided into (at least) two sub-populations, although the origin of these remains unclear. In the Milky Way and M31, the metal-poor globular cluster sub-populations display halo-like kinematics and spatial distributions (e.g. Zinn 1985; Ashman \& Zepf 1998; Barmby et al. 2000; Perrett et al. 2002), while the metal-rich globular clusters may be associated with the bulge and/or thick disk (e.g. Minniti 1995; Barbuy et al. 1998; Côté 1999; Bica et al. 2006). The globular cluster sub-populations in elliptical galaxies show many similarities to those in spirals, and some of the metal-rich clusters may have formed in galaxy mergers (e.g. Ashman \& Zepf 1992). Some of the metal-poor ("halo") clusters may have been accreted from dwarf galaxies (Da Costa \& Armandroff $1995)$ or in proto-galactic fragments from which the halo assembled (Searle \& Zinn 1978). However, there is evidence based on metallicity that globular clusters could not have been formed from the destruction of dwarf galaxies (see Koch et al. 2008, and references there-in). A major challenge is to establish how each

\footnotetext{
* Based on data collected at the Cerro Paranal, Chile run by the European Southern Observatory (ESO) under programme ID 077.D0403(A) and 077.D-0403(B).

$\star \star$ Table 6 and Fig. 6 are only available in electronic form at

http://www. aanda.org
}

one of these mechanisms may fit into the paradigm of hierarchical structure formation (e.g. Santos 2003).

One important step towards understanding the roles of merging and accretion processes is to extend our knowledge about globular clusters to many different galaxy types, such as dwarf galaxies and late-type spirals, in a range of environments. Studies of globular clusters in spiral galaxies are more difficult than in early-type galaxies because the globular cluster systems are generally poorer and appear superposed on an irregular background. Consequently, most studies of extragalactic globular clusters have focused on elliptical galaxies.

In spite of the similarities, there are also important differences between globular cluster systems of large ellipticals and those of spirals like the Milky Way. Elliptical galaxies generally have many more globular clusters per unit host galaxy luminosity (i.e., higher globular cluster specific frequencies Harris \& van den Bergh (1981) than spirals), and on average the globular cluster systems of ellipticals are more metal-rich (Kissler-Patig et al. 1999). The best studied globular cluster systems in spiral galaxies are those associated with the Milky Way and M 31. Globular clusters in M31 appear similar to those in the Milky Way in terms of their luminosity functions, metallicities and, size distributions (Crampton et al. 1985; Perrett et al. 2002; Barmby et al. 2002). M 33 has a large number of star clusters (Christian \& Schommer 1982, 1988; Chandar et al. 2001) but many of them have young ages and there may be only a dozen or so truly "halo" globular clusters (Sarajedini et al. 2000). The Magellanic Clouds are also well-known for their rich 
cluster systems, but again only few of these are truly old globular clusters. The Large Magellanic Cloud has about 13 old globular clusters (Johnson et al. 1999), which however show disk-like kinematics. The Small Magellanic Cloud has only one old globular cluster, NGC 121.

The globular cluster populations in low-luminosity spirals are almost unknown. The question of how these clusters formed (and their host galaxy) remain unanswered. Considering that these kind of galaxies remain almost unperturbed during their live, it is probable that we are observing their first population of globular clusters and therefore, we can approach to the conditions in which the host galaxies were formed.

The nearby Sculptor group hosts several late-type galaxies whose globular cluster systems are potentially within reach of spectroscopic observations with $8 \mathrm{~m}$ telescopes in a few hours of integration time. A previous study of the star cluster population in the Sculptor group was done by Olsen et al. (2004). They observed several globular clusters candidates, finding 19 globular clusters in four galaxies, most of them metal-poor with $[\alpha / \mathrm{Fe}]$ lower than the Milky Way globular clusters.

In this paper we concentrate on the late-type, low-luminosity spiral galaxy NGC 45 in Sculptor, in which we have previously identified a surprisingly rich population of old globular cluster candidates in HST/ACS imaging. NGC 45 is classified as a low-luminosity spiral galaxy with $B=11.37 \pm 0.11$ and $B-V=0.71$ (Paturel et al. 2003). It is located at $\sim 5 \mathrm{Mpc}$ from us, $(m-M)_{0}=28.42 \pm 0.41$ (Bottinelli et al. 1985), in the periphery of the Sculptor group. In Mora et al. (2007) we found 19 globular clusters located in projection with the galaxy bulge, which appear to belong to the metal-poor population. Those 19 globular clusters yield a $S_{N}$ of 1.4-1.9, which is high for a late-type galaxy.

In this paper we focus on 8 of those 19 globular clusters. We analyze them through spectroscopy to confirm or reject their globular cluster status and to constraint ages and metallicities.

\section{Candidates selection, observation and reductions}

\subsection{Globular cluster selection}

In Mora et al. (2007) we identified cluster candidates as extended objects, using a variety of size selection criteria based on the BAOLAB/ISHAPE (Larsen 1999) and SExtractor (Bertin \& Arnouts 1996) packages. We found 19 extended objects fulfilling the color criteria $0.8<V-I<1.2$, with magnitudes $V=19.5$ up to $V=22.5$ that were interpreted as globular clusters. The detected globular cluster candidates had a mean color of $V-I=0.90$, consistent with a metal-poor, old population. The mean half-light radius of the globular cluster candidates was $R_{\text {eff }}=2.9 \pm 0.7$ pc (error is the standard error of the mean), similar to that of globular clusters in other galaxies.

\subsection{Observations and reductions}

The spectra were acquired in service mode on the ESO period 77 using the ESO Focal Reducer/low dispersion Spectrograph (FORS2) through the Multi-object spectroscopy mask exchange unit (MXU), which is mounted in Kueyen/UT2 VLT telescope at Cerro Paranal Chile. We used the GRIS_600B+22 which has a wavelength range from $3330 \AA$ up to $6210 \AA$ with a dispersion at the central wavelength ( $4650 \AA$ ) of $0.75 \AA /$ pixel. Because of the globular cluster positions, we were only able to place 8 globular clusters on the MXU. Extra 16 filler slits were placed on young

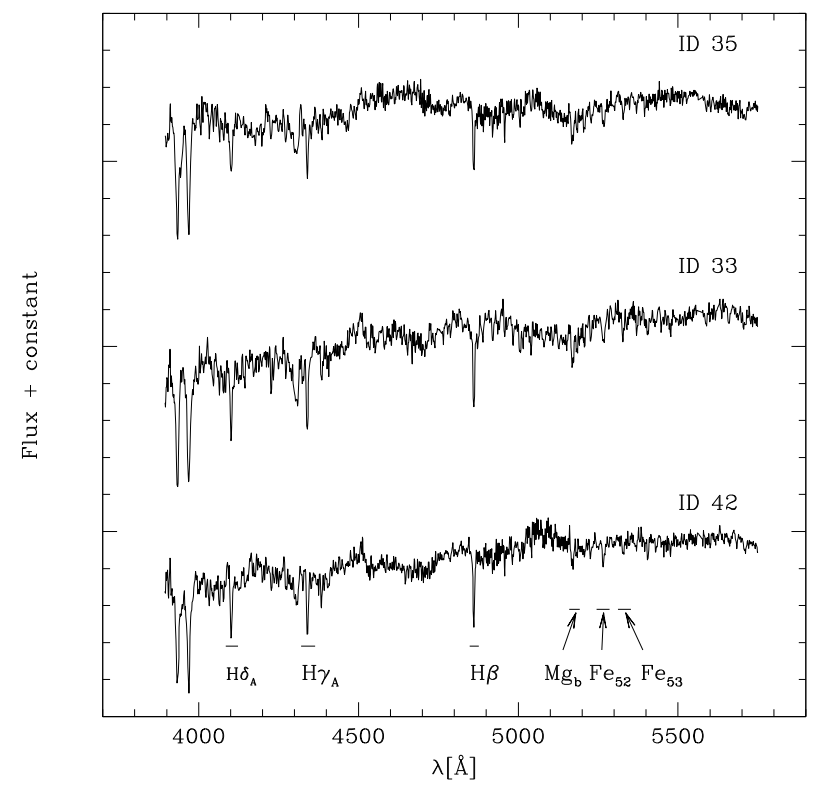

Fig. 1. Samples of the spectra. The spectra have been shifted to the 0 radial velocity and an offset in flux has been added for best clarity of the sample. Some Lick-index passband are indicated at the bottom of the panel.

Table 1. Log of the observations.

\begin{tabular}{lccc}
\hline \hline Object & Mode & N of exposures $\times$ time & Obs type \\
\hline NGC 45 & MXU & $17 \times(3 \times 1200 \mathrm{~s})$ & Science \\
NGC 45 & MXU & $2 \times 1095 \mathrm{~s}$ & Science \\
HD 180928 & LSS & $3 \times 1.00 \mathrm{~s}$ & Lick std \\
HD 195633 & LSS & $3 \times 1.00 \mathrm{~s}$ & Lick std \\
HD 165195 & LSS & $2 \times(3 \times 3.00 \mathrm{~s})$ & Lick std \\
HD 003567 & LSS & $3 \times 5.00 \mathrm{~s}$ & Lick std \\
HD 221148 & LSS & $3 \times 1.00 \mathrm{~s}$ & Lick std \\
HD 006695 & LSS & $2 \times(3 \times 0.63 \mathrm{~s})$ & Lick std \\
\hline
\end{tabular}

The second column indicates the observed mode: multi-object spectroscopy mask exchange unit (MXU) and Long slit spectroscopy (LSS).

star regions, the galaxy bulge, the sky and one star. On each object a slit of $1.0^{\prime \prime}$ width was placed and MXU observations were done in 7 Observing Blocks (OBs). A sample of these spectra are shown in Fig. 1. For the calibration, we acquired 6 Lick/IDS standard stars from Worthey et al. (1994) in long slit spectra mode using the same configuration of the globular cluster observations (for a log of the observations see Table 1). The six standard stars were selected according to their spectral type (i.e. K3III, G0V, G5IV, F5VI, K3IIIV and, A3V), covering the range of spectral types expected in a globular cluster. Each standard was observed in 3 exposure sets with a small offset in the direction of the slit in order to avoid bad pixels in the final combined spectra.

The spectra (science and standard stars) were reduced (i.e. bias subtracted, flat field corrected, optical distortion corrected and, wavelength calibrated) using the ESO Recipe Execution Tool v3.6 (ESO-REX) ${ }^{1}$. Typical rms from the distortion corrections were on the order of 0.4 pixels and the wavelength calibration accuracy of the model applied during the wavelength calibration was on the order of $0.08 \AA$.

\footnotetext{
1 http://www.eso.org/sci/data-processing/software/ cpl/esorex.html
} 

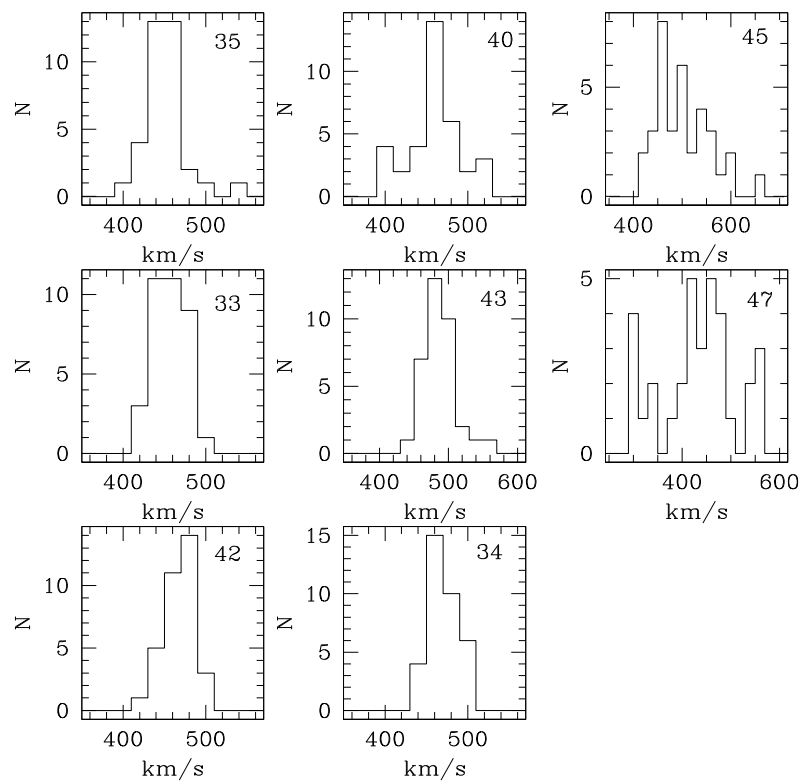

$\mathrm{km} / \mathrm{s}$

Fig. 2. Velocity distributions of the globular clusters. The bin size is $20 \mathrm{~km} \mathrm{~s}^{-1}$. The number on each pannel corresponds to the globular cluster ID.

In the following section we explain the radial velocity measurements. We only focus on the globular clusters because filler spectra were too faint to have reasonable radial velocity measurements.

\section{Radial velocities}

Radial velocities were first derived for the standard stars. Since each standard star was acquired in a set of 3 consecutive exposures, we derived the radial velocity on each single exposure. This was accomplished by cross correlating a zero velocity elliptical galaxy template from Quintana et al. (1996) with each spectrum using the FXCOR IRAF ${ }^{2}$ task. Each single spectrum was shifted to zero radial velocity and combined into a high signal to noise standard star spectrum.

Globular cluster spectra taken in the same OB were combined, yielding 7 spectra for each globular cluster. Each one of them, in combination with the high signal to noise standard star spectra, were used to derive the globular cluster velocities through cross correlation using the FXCOR IRAF task. On each cross correlation we select a region of $200 \AA$ width centered in the $\mathrm{Ca}$ II $\mathrm{H}+\mathrm{K}, \mathrm{H} \beta$ and, $\mathrm{H} \gamma$ features. An extra region from $5000 \AA$ up to $5500 \AA$ was also considered for cross correlation. We note that the cross correlations between standard-star types $\mathrm{A}$ and $\mathrm{K}$; and the globular cluster spectra were particularly difficult, most likely because such stars provide a poorer match to the overall spectrum of a globular cluster. This effect, combined with the low signal-to-noise of the spectra (especially for the globular clusters ID 45 and ID 47) caused the velocity measurements to be more uncertain when based on these stars.

In Fig. 2 we show histograms of all the individual velocity measurements for each cluster. We adopted the average of the distribution as the final velocity value of each globular cluster. The error was obtained from the standard deviation divided by

${ }^{2}$ IRAF is distributed by the National Optical Astronomical Observatory, which is operated by the Association of Universities for Research in Astronomy, Inc, under cooperative agreement with the National Science Foundation.

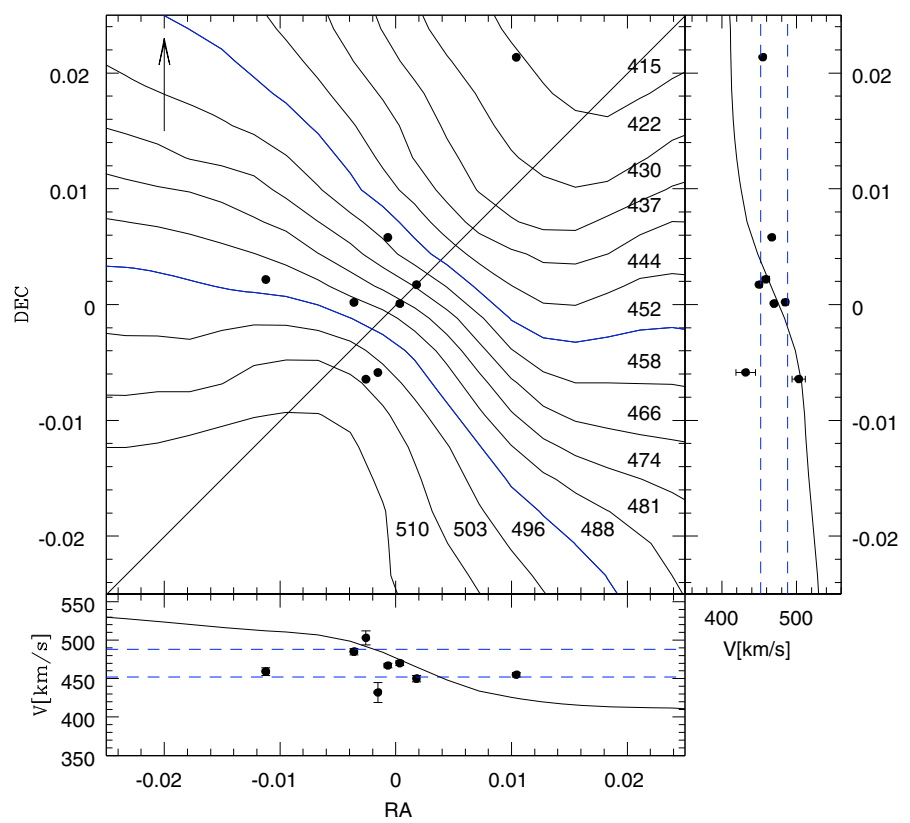

Fig. 3. Main panel: position of the bona fide globular clusters with overplotted isovelocity contours from Chemin et al. (2006). Arrow indicates the north and East is on the left. The sub panels on the bottom as well as on the right show the globular cluster velocity projections of the main panel as function of RA and Dec respectively. Blue lines indicate isovelocity lines for $480 \mathrm{~km} \mathrm{~s}^{-1}$ and $452 \mathrm{~km} \mathrm{~s}^{-1}$. The straight line crossing the main panel has the purpose of illustrating the velocity changes when it is projected as function of RA and Dec on the sub panels. Numbers next to the isovelocity contours indicate the velocity in $\mathrm{km} \mathrm{s}^{-1}$.

Table 2. Derived velocities of the globular clusters.

\begin{tabular}{cccc}
\hline \hline ID & Vel $\left(\mathrm{km} \mathrm{s}^{-1}\right)$ & $\sigma$ & $N$ \\
\hline 33 & $455 \pm 3$ & 19 & 37 \\
34 & $470 \pm 3$ & 17 & 35 \\
35 & $450 \pm 4$ & 25 & 35 \\
40 & $459 \pm 5$ & 32 & 35 \\
42 & $467 \pm 3$ & 17 & 34 \\
43 & $485 \pm 4$ & 23 & 35 \\
45 & $503 \pm 9$ & 55 & 33 \\
47 & $432 \pm 13$ & 77 & 33
\end{tabular}

$\sigma$ is the standard deviation and $N$ corresponds to the number of measurements.

the square root of the number of measurements. Values are listed in Table 2.

In Fig. 3 we show the position of the globular clusters with overplotted isovelocity contours from Chemin et al. (2006). The sub-panel on the bottom and the sub-panel on the right shows the projected velocity as function of RA and Dec. The panels show that our velocity measurements are consistent with no overall rotation of the globular cluster system. Globular clusters located near the center of the galaxy show velocities consistent with the observed H I gas velocities from Chemin et al. (2006). The greatest difference between the globular cluster velocities was $\Delta V=71 \pm 16 \mathrm{~km} \mathrm{~s}^{-1}$. It corresponds to the difference of velocity between the globular cluster ID $=45$ and 47, which also show the largest errors. Therefore, globular cluster velocities are mainly concentrated between $V=430-480 \mathrm{~km} \mathrm{~s}^{-1}$, as is seen in Fig. 3.

The velocities of the GCs clearly do not match the isovelocity contours of Chemin et al. (2006), and we thus exclude that 
the GCs are associated with the disk component of NGC 45. The velocity dispersion is $\sigma=20 \pm 4 \mathrm{~km} \mathrm{~s}^{-1}$, which is significantly larger than the measurement errors (Table 2) and smaller than other similar galaxies like M 33 (Chandar et al. (2002) found values of $\sigma=54 \pm 8 \mathrm{~km} \mathrm{~s}^{-1}$ for disk/bulge globular clusters and $\sigma=83 \pm 13 \mathrm{~km} \mathrm{~s}^{-1}$ for halo globular clusters). Therefore, we conclude that the globular cluster velocities in NGC 45 are indicative of halo- or bulge like kinematics, with little or no overall rotation.

\section{Lick index calibrations}

In the following we estimate abundances for our globular cluster sample using the Lick/IDS system of absorption line indices. Passband definitions were taken from G. Worthey's web page ${ }^{3}$ which corresponds to Trager et al. (1998) for the new wavelength definitions, Worthey et al. (1994) for the old definitions including $\mathrm{H}_{\delta}$ and $\mathrm{H}_{\gamma}$ definitions from Worthey \& Ottaviani (1997).

The Lick/IDS system is designed to measure absorption features such as $\mathrm{CN}, \mathrm{H} \beta, \mathrm{Fe}, \mathrm{Mg}, \mathrm{G}$ (molecular bands) and blend of absorption lines present in old populations. These features were used to construct a library from several stars observed at the Lick observatory. Six of these standard stars were taken during the observations that are presented in this work. Due to our telescope configuration, the standard stars spectra, as well as the globular cluster spectra, have higher spectral resolution than the original Lick/IDS system. Thus, we must carefully degrade our spectra in order to match the Lick/IDS spectral resolution. One way to quantify the difference between our instrumental system and the original Lick/IDS spectra is to measure the $F W H M$ of narrow spectral features. We measured the $F W H M$ of the sky lines in our sky spectra and we found a typical $F W H M=4.9 \AA$. This value was used as input for the code used for the index derivations.

The indices were measured using the GONZO code from Puzia et al. (2002). Briefly, GONZO degrades the spectra to the Lick/IDS index system with a wavelength-dependent Gaussian kernel in order to match the resolution from Worthey \& Ottaviani (1997). GONZO also derives the uncertainties of the indices by considering the Poisson statistics from the error spectra. These statistics are used in addition to the random noise when creating artificial science spectra and measuring the indices on these. For further details on GONZO, see Puzia et al. (2002).

In the comparisons between our standard stars and the Lick/IDS system, we assumed that the transformation between our measured Lick/IDS indices and the standard values was linear with a slope of unity, so that only an offset is needed to match the standard Lick/IDS system. Also we avoided possible changes of the slopes due to outlier measurements (which were impossible to identify because of the small number of the observed standard stars).

The zero-point offsets are given in Table 3 and a comparison between our measurements and the Lick/IDS system is shown in Fig. 6. In Table $3 \mathrm{ZP}$ corresponds to the zero point needed in order to match the Lick/IDS system and $\sigma$ corresponds to the error which was calculated considering the standard deviation divided by the square root of the number of measurements. The reader may note that we do not list values for the $\mathrm{TiO}$ indices because the $\mathrm{TiO}$ indices were outside the wavelength coverage.

${ }^{3}$ http://astro.wsu.edu/worthey/html/index.table.html
Table 3. Calibration summary of the Lick Indices.

\begin{tabular}{lrrc}
\hline \hline Index & ZP & $\sigma$ & Units \\
\hline $\mathrm{CN}_{1}$ & 0.0301 & 0.0123 & $\mathrm{mag}$ \\
$\mathrm{CN}_{2}$ & 0.0364 & 0.0087 & $\mathrm{mag}$ \\
$\mathrm{Ca} 4227$ & 0.3878 & 0.0830 & $\AA$ \\
$\mathrm{G} 4300$ & 0.8417 & 0.2467 & $\AA$ \\
$\mathrm{Fe} 4383$ & 0.6260 & 0.2155 & $\AA$ \\
$\mathrm{Ca} 4455$ & 0.1553 & 0.1183 & $\AA$ \\
$\mathrm{Fe} 4531$ & 0.3835 & 0.2359 & $\AA$ \\
$\mathrm{Fe} 4668$ & -0.0715 & 0.2840 & $\AA$ \\
$\mathrm{H} \beta$ & 0.0515 & 0.1234 & $\AA$ \\
$\mathrm{Fe} 5015$ & 0.0583 & 0.2394 & $\AA$ \\
$\mathrm{Mg}$ & -0.0116 & 0.0034 & $\mathrm{mag}$ \\
$\mathrm{Mg} g_{2}$ & -0.0149 & 0.0062 & $\mathrm{mag}$ \\
$\mathrm{Mg}$ & 0.2932 & 0.0501 & $\AA$ \\
$\mathrm{Fe} 5270$ & 0.4598 & 0.0563 & $\AA$ \\
$\mathrm{Fe} 5335$ & 0.2400 & 0.1041 & $\AA$ \\
$\mathrm{Fe} 5406$ & 0.0729 & 0.0581 & $\AA$ \\
$\mathrm{Fe} 5709$ & 0.1342 & 0.0667 & $\AA$ \\
$\mathrm{TiO}_{1}$ & - & - & $\mathrm{mag}$ \\
$\mathrm{TiO}_{2}$ & - & - & $\mathrm{mag}$ \\
$\mathrm{H} \delta_{A}$ & 0.1376 & 0.3270 & $\AA$ \\
$\mathrm{H} \gamma_{A}$ & -0.4629 & 0.3295 & $\AA$ \\
$\mathrm{H} \delta_{F}$ & 0.6445 & 0.1504 & $\AA$ \\
$\mathrm{H} \gamma_{F}$ & -0.1960 & 0.1333 & $\AA$ \\
\hline & & &
\end{tabular}

\section{Results}

\subsection{Age diagnostic plots}

In this subsection we discuss the results of the indices measurements and their comparisons with $\alpha / \mathrm{Fe}$-enhanced models from Thomas et al. (2003) and Thomas et al. (2004). We adopted the index $[\mathrm{MgFe}]^{\prime}=\sqrt{\mathrm{Mgb} \times(0.72 \times \mathrm{Fe} 5270+0.28 \times \mathrm{Fe} 5335)}$ which is $[\alpha / \mathrm{Fe}]$ independent defined by Thomas et al. (2003). We also adopted from González (1993) the $\langle\mathrm{Fe}\rangle=(\mathrm{Fe} 5270+$ Fe5335)/2 index definition. Figure 4 show the age metallicity diagnostic plots for the Balmer line indices $\mathrm{H} \beta, \mathrm{H} \gamma_{A}, \mathrm{H} \gamma_{F}$, $\mathrm{H} \delta_{A}$ and, $\mathrm{H} \delta_{F}$ against $[\mathrm{MgFe}]^{\prime}$. All our globular clusters show $[\mathrm{MgFe}]^{\prime}$ values less than or equal to $2 \AA$, which makes them metal poor.

Ages are poorly constrained and depend on the Balmer line used for the comparison with models, but the metallicities are consistently sub-solar with $[\mathrm{Z} / \mathrm{H}]<-0.33$. In the left panel of Fig. 5 we show the average iron versus $\mathrm{Mg} 2$ over-plotted with models from Thomas et al. (2004) for $[\alpha / \mathrm{Fe}]=-0.3,0.0$ and, 0.3 ; and ages of 5 and 15 Gyr. The panel shows globular clusters located in the sub-solar metallicity region with values lower than $[\mathrm{Z} / \mathrm{H}]=-0.33$ corroborating the same result of Fig. 4 and, despite the great uncertainties, the globular clusters seem to be better described by models with sub-solar $[\alpha / \mathrm{Fe}]=0.0,-0.3$ element ratios. This conclusion becomes less strong when the two Fe index are plotted separately versus $\mathrm{Mgb}$ as it is seen in the central and right panels of Fig. 5. For the $\mathrm{Fe}_{5270}$ index, globular clusters show $[\alpha / \mathrm{Fe}] \leq 0.0$ while for the $\mathrm{Fe}_{5335}$ index, globular clusters are scattered between $-0.3<[\alpha / \mathrm{Fe}]<0.3$. The derived values of $[\alpha / \mathrm{Fe}]$ element ratios and metallicities seems to be consistent with values found by Olsen et al. (2004) in 4 Sculptor galaxies. They concluded that all globular clusters in the Sculptor group have $[\mathrm{Fe} / \mathrm{H}] \leq-1.0$ (we found values for $\mathrm{NGC} 45[\mathrm{Fe} / \mathrm{H}] \leq-0.3)$ and values of $-0.3 \pm 0.15<[\alpha / \mathrm{Fe}]<$ $0.0 \pm 0.15$ for the measured globular clusters. 

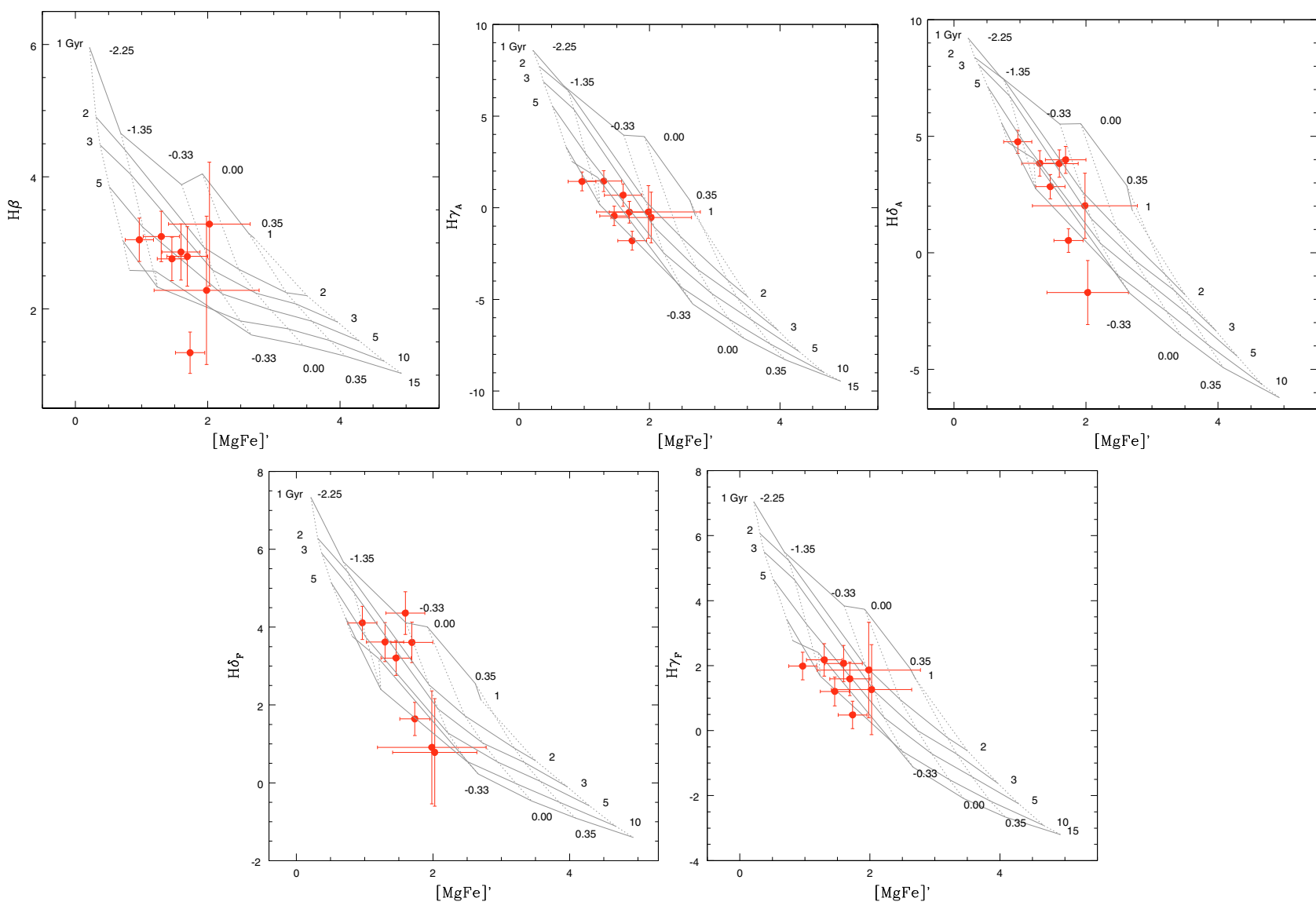

Fig. 4. Age diagnostic plots. The over-plotted grid corresponds to SSP model from Thomas et al. (2004) for $[\alpha / \mathrm{Fe}]=0.0$. Doted lines correspond to metallicities $[\mathrm{Z} / \mathrm{H}]=-2.25,-1.35,-0.33,0.0,0.35$ and, 0.67 dex. Solid lines correspond to ages of 1, 2, 3, 5, 10 and 15 Gyr.
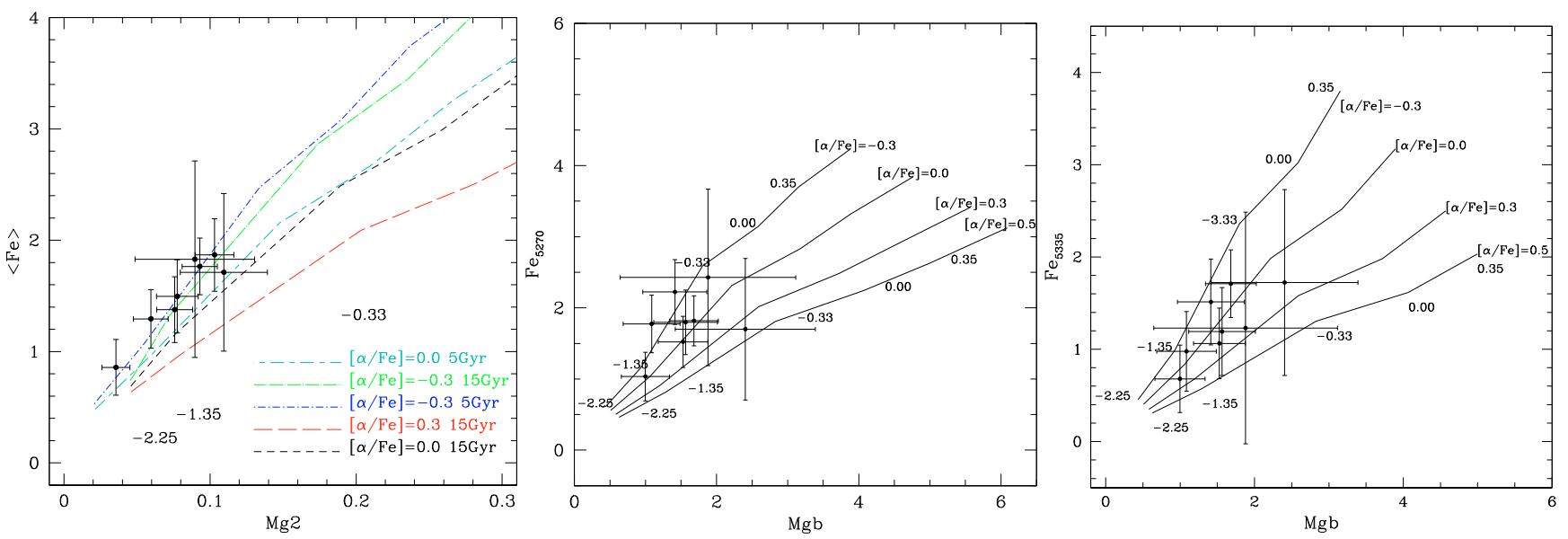

Fig. 5. Left panel: iron $\langle\mathrm{Fe}\rangle$ as function of $\mathrm{Mg}$ 2. Central panel: $\mathrm{Fe}_{5278}$ index versus Mgb. Right panel: $\mathrm{Fe}_{5335}$ index versus Mgb.

\subsection{Comparison with photometric ages}

In spite of the uncertainties of the star cluster metallicities and ages, it is worth to comment how ages and metallicities derived here compare with the previous ages and metallicities assumed in Mora et al. (2007). As a reminder, in Mora et al. (2007) we used GALEV models (Anders \& Fritze-v. Alvensleben 2003) considering 4 metallicites: $Z=0.004,0.008,0.02$, and 0.05 which are equivalent to $[\mathrm{Fe} / \mathrm{H}]=-0.7,0.4,0$, and +0.4 . In the present work, we have 3 globular clusters $($ ID $=33,34$ and 35 ) in common with our previous work.
In Table 4 we show the $[Z / H]$ values calculated for each index and its mean value calculated from the age diagnostic plots. We do not extrapolate ages nor metallicities of globular clusters lying outside the model grids. Errors were calculated considering the highest and lowest values for each index within the error bars. Also, if the error bar (or a part of it) lie outside the model grids, we do not extrapolate its value and we considered the farest point of the grid as the maximum (or minimum) error value. Therefore, errors lie within the grid values. Globular clusters where this happened are shown in boldface on the Table 4. We found that the derived metallicities agree on each index and 
Table 4. Metallicities from age diagnostic plots.

\begin{tabular}{|c|c|c|c|c|c|c|}
\hline $\begin{array}{l}\text { (1) } \\
\text { ID }\end{array}$ & $\begin{array}{c}(2) \\
Z / H\left(\mathrm{H}_{\beta}\right)\end{array}$ & $\begin{array}{c}\text { (3) } \\
Z / H\left(\mathrm{H} \gamma_{\mathrm{A}}\right)\end{array}$ & $\begin{array}{c}(4) \\
Z / H\left(\mathrm{H} \delta_{\mathrm{F}}\right)\end{array}$ & $\begin{array}{c}(5) \\
Z / H\left(\mathrm{H} \delta_{\mathrm{A}}\right) \\
\end{array}$ & $\begin{array}{c}(6) \\
Z / H\left(\mathrm{H} \gamma_{\mathrm{F}}\right)\end{array}$ & $\begin{array}{c}(7) \\
\langle[Z / H]\rangle\end{array}$ \\
\hline 33 & $-1.1_{-0.55}^{+0.40}$ & $-1.15_{-0.60}^{+0.45}$ & $-1.0_{-0.75}^{+0.65}$ & & $-1.2_{-0.50}^{+0.40}$ & $-1.12_{-0.59}^{+0.5}$ \\
\hline 34 & - & -0.9 & - & 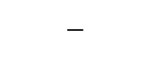 & _ & $-0.9_{-0.1}^{+0.2}$ \\
\hline 35 & -0.85 & -0.8 & - & & $0.85_{-}^{+}$ & $-0.79_{-0.46}^{+0.36}$ \\
\hline 40 & -0.65 & - & & & - & \\
\hline 42 & $-1.0_{-0.35}^{+0.35}$ & $-1.15_{-0.15}^{+0.35}$ & $-0.9_{-0.45}^{+0.55}$ & $-1.05_{-0}^{+0}$ & - & $-1.03_{-0.31}^{+0.41}$ \\
\hline 43 & $-0.75_{-0.45}^{+0.40}$ & $-0.8_{-0.40}^{+0.40}$ & $-0.4_{-0.60}^{+0.30}$ & $-0.45_{-0.60}^{+0.30}$ & $-0.8_{-0.4}^{+0.4}$ & $-0.64_{-0.49}^{+0.36}$ \\
\hline 45 & $-0.15_{-1.05}^{+0.65}$ & $-0.35_{-0.85}^{+0.95}$ & - & - & $-0.4_{-0.80}^{+0.70}$ & $-0.30_{-0.9}^{+0.76}$ \\
\hline 47 & $-0.6_{-0.80}^{+0.65}$ & $-0.35_{-1.05}^{+0.60}$ & - & $-0.35_{-1.00}^{+0.55}$ & $-0.3_{-1.10}^{+0.55}$ & $-0.40_{-0.99}^{+0.59}$ \\
\hline
\end{tabular}

Column (1): globular cluster ID. Columns (2) to (6): $Z / H$. The index used for its derivation is shown between brackets. Column (7): average $Z / H$.

Table 5. Derived ages in Gyr from age diagnostic plots.

\begin{tabular}{|c|c|c|c|c|c|c|c|c|}
\hline (1) & (2) & (3) & (4) & (5) & (6) & (7) & (8) & (9) \\
\hline ID & $\operatorname{Age}\left(\mathrm{H}_{\beta}\right)$ & $\operatorname{Age}\left(\mathrm{H} \gamma_{A}\right)$ & $\operatorname{Age}\left(\mathrm{H} \delta_{\mathrm{F}}\right)$ & $\operatorname{Age}\left(\mathrm{H} \delta_{\mathrm{A}}\right)$ & $\operatorname{Age}\left(\mathrm{H} \gamma_{\mathrm{F}}\right)$ & $\langle$ Age $\rangle$ & $\log (\mathrm{Age} / \mathrm{yr})$ & Age \\
\hline 33 & $4.7_{-2.3}^{+4.4}$ & $\begin{array}{l}5.8_{-3.2}^{+9.2} \\
\end{array}$ & $3.5_{-2.5}^{+10.0}$ & $5.4_{-3.7}^{+8.8}$ & $7.2_{-3.6}^{+6.3}$ & $5.32_{-3.06}^{+7.74}$ & $9.06_{-1.14}^{+0.39}$ & $1.14_{-1.06}^{+1.67}$ \\
\hline 34 & - & $9.9_{-3.1}^{+0.8}$ & - & - & - & $9.90_{-3.1}^{+0.80}$ & $9.10_{-0.65}^{+0.91}$ & $1.25_{-0.97}^{+8.97}$ \\
\hline 35 & $4.6_{-2.9}^{+4.5}$ & $4.3_{-2.1}^{+10.4}$ & - & $2.3_{-0.9}^{+5.1}$ & $4.6_{-2.8}^{+9.0}$ & $4.52_{-1.76}^{+6.09}$ & $6.91_{-0.09}^{+1.43}$ & $0.008_{-0.02}^{+0.27}$ \\
\hline 40 & $7.0_{-2.9}^{+6.4}$ & - & $4.5_{-2.8}^{+10.5}$ & $6.9_{-4.0}^{+8.1}$ & - & $6.13_{-3.23}^{+8.30}$ & - & - \\
\hline $\begin{array}{l}42 \\
43\end{array}$ & $\begin{array}{l}5.9_{-3.0}^{+9.1} \\
4.4_{-2.5}^{+5.1}\end{array}$ & $\begin{array}{l}9.5_{-4.2}^{+3.6} \\
5.6_{-3.3}^{+8.5}\end{array}$ & $\begin{array}{c}\mathbf{3 . 8}_{-2.5}^{+\mathbf{1 1 . 2}} \\
1.4_{-0.4}^{+3.2}\end{array}$ & $\begin{array}{l}\mathbf{6 . 9} \\
1.3 .9 \\
1.7_{-0.4}^{+3.9}\end{array}$ & $\begin{array}{c}- \\
5.5_{-3.4}^{+8.4}\end{array}$ & $\begin{array}{l}6.52_{-3.4}^{+7.95} \\
3.72_{-2}^{+5.78}\end{array}$ & $\begin{array}{l}- \\
-\end{array}$ & - \\
\hline 45 & $1.6_{-0.6}^{+7.9}$ & $2.7_{-1.7}^{+10.8}$ & - & & $3.4_{-2.4}^{+8.5}$ & $2.56_{-1.57}^{+9.06}$ & - & - \\
\hline 47 & $6.9_{-5.2}^{+8.1}$ & $2.5_{-1.3}^{+11.3}$ & - & $2.6_{-1.3}^{+7.4}$ & $2.0_{-1.0}^{+11}$ & $3.50_{-2.2}^{+9.45}$ & - & - \\
\hline
\end{tabular}

Column (1): globular cluster ID. Columns (2) to (6): derived ages. The index used for its derivation is shown between brackets. Column (7): average age. Columns (8) and (9): ages derived from GALEV.

we concluded that the most adequate metallicity for age and mass derivations with photometry is $Z=0.004$ of the GALEV models.

In Table 5 we show individual ages derived from the age diagnostic plots for each Balmer index, and the mean value considering all Balmer indices. We do not extrapolated values outside the model grids and errors were calculated in the same way as we did in Table 4. Values in boldface indicate that the errors lie outside the model grid. The last two columns show the derived ages from GALEV (considering $Z=0.004$ ). Ages derived from photometry do not agree with the values derived using spectroscopy. Photometric ages were underestimated, compared with the spectroscopic ones. This underestimation is more dramatic for the globular cluster ID $=35$ where the photometric age do not share the same order of magnitude as the spectroscopic ones. This result is not entirely unexpected, since old globular clusters are faint in the $U$-band, which provides much of the leverage for age determinations. Furthermore, model uncertainties and degeneracies in age/metallicity/reddening all combine to produce larger uncertainties on the photometric ages for old globular clusters.

\section{Discussion and conclusion}

Although uncertainties on the age estimates for our sample of globular clusters in NGC 45 remain large, we were able to constrain their metallicities and $\alpha / \mathrm{Fe}$ abundance ratios. These showed that the globular clusters in NGC 45 are metal poor, corroborating the metal poor population deducted from the globular cluster colors in Mora et al. (2007).

Assuming that the globular clusters are tracer of star formation events, considering that NGC 45 is an isolated galaxy, probably a background object and, not a true member of the Sculptor group (Puche \& Carignan 1988), it is puzzling how these entities formed in this galaxy. Whiting (1999) suggested that NGC 45 once made a close pass by the Sculptor group, getting close to NGC 7793 and transferring angular momentum. This would have been excited the globular cluster formation in NGC 45 but there is no record of this in the globular cluster population. Therefore, the formation of the globular clusters must be happen at early times probably when the galaxy assembled.

The globular cluster velocities and the velocity dispersion of the system argue in favor of a real association between the 
globular clusters and the galaxy bulge. The velocity distribution of the GC population seems to be dominated by random motions, although it would be desirable to corroborate this statement with further velocity measurements of the remaining 11 candidates.

The sub-solar $[\alpha / \mathrm{Fe}]$ values derived here are unlike those typically observed in old GC populations (e.g. Puzia et al. 2005), including those in the Milky Way. However, we note that they are consistent with those derived by Olsen et al. (2004). It is also of interest to note that dwarf galaxies in the Local Group tend to show less alpha-enhanced abundance ratios than the Milky Way (Sbordone et al. 2005; Tolstoy et al. 2003), despite the fact that many of them are satellite galaxies (e.g. LMC, SMC, etc.). This may point to important differences in the early chemical evolution in these different types of galaxies, and is potentially an argument against the notion that a major fraction of the GCs in large galaxies could have been accreted from minor galaxies similar to those observed today.

One possible explanation for the relatively low $[\alpha / \mathrm{Fe}]$ ratios of the GCs in NGC 45 is that the formation and assembly of the halo/bulge component took longer than in major galaxies like the Milky Way. This would allow time for Type Ia supernovae to appear and enrich the gas with greater amounts of Fe.

Acknowledgements. We would like to thank Thomas Puzia for providing us the GONZO code, Steffen Mieske for providing us the elliptical galaxy spectra template, Laurent Chemin for gently provide us the NGC $45 \mathrm{H}$ I velocity field data, and the referee for his/her work.

\section{References}

Anders, P., \& Fritze-v. Alvensleben, U. 2003, A\&A, 401, 1063

Ashman, K. M., \& Zepf, S. E. 1992, ApJ, 384, 50

Ashman, K. M., \& Zepf, S. E. 1998, Globular Cluster Systems (Globular cluster systems / Keith M. Ashman, Stephen E. Zepf. Cambridge, UK; New York: Cambridge University Press)

Barbuy, B., Bica, E., \& Ortolani, S. 1998, A\&A, 333, 117

Barmby, P., Huchra, J. P., Brodie, J. P., et al. 2000, AJ, 119, 727

Barmby, P., Perrett, K. M., \& Bridges, T. J. 2002, MNRAS, 329, 461
Bertin, E., \& Arnouts, S. 1996, A\&AS, 117, 393

Bica, E., Bonatto, C., Barbuy, B., \& Ortolani, S. 2006, A\&A, 450, 105

Bottinelli, L., Gouguenheim, L., Paturel, G., \& de Vaucouleurs, G. 1985, ApJS, 59, 293

Chandar, R., Bianchi, L., \& Ford, H. C. 2001, A\&A, 366, 498

Chandar, R., Bianchi, L., Ford, H. C., \& Sarajedini, A. 2002, ApJ, 564, 712

Chemin, L., Carignan, C., Drouin, N., \& Freeman, K. C. 2006, AJ, 132, 2527

Christian, C. A., \& Schommer, R. A. 1982, ApJS, 49, 405

Christian, C. A., \& Schommer, R. A. 1988, AJ, 95, 704

Côté, P. 1999, AJ, 118, 406

Crampton, D., Cowley, A. P., Schade, D., \& Chayer, P. 1985, ApJ, 288, 494

Da Costa, G. S., \& Armandroff, T. E. 1995, AJ, 109, 2533

González, J. 1993, Ph.D. Thesis (Univ. of Califormia)

Harris, W. E., \& van den Bergh, S. 1981, AJ, 86, 1627

Johnson, J. A., Bolte, M., Stetson, P. B., Hesser, J. E., \& Somerville, R. S. 1999, ApJ, 527, 199

Kissler-Patig, M., Ashman, K. M., Zepf, S. E., \& Freeman, K. C. 1999, AJ, 118, 197

Koch, A., Grebel, E. K., Gilmore, G. F., et al. 2008, AJ, 135, 1580

Larsen, S. S. 1999, A\&AS, 139, 393

Minniti, D. 1995, AJ, 109, 1663

Mora, M. D., Larsen, S. S., \& Kissler-Patig, M. 2007, A\&A, 464, 495

Olsen, K. A. G., Miller, B. W., Suntzeff, N. B., Schommer, R. A., \& Bright, J. 2004, AJ, 127, 2674

Paturel, G., Petit, C., Prugniel, P., et al. 2003, A\&A, 412, 45

Perrett, K. M., Bridges, T. J., Hanes, D. A., et al. 2002, AJ, 123, 2490

Puche, D., \& Carignan, C. 1988, AJ, 95, 1025

Puzia, T. H., Saglia, R. P., Kissler-Patig, M., et al. 2002, A\&A, 395, 45

Puzia, T. H., Kissler-Patig, M., Thomas, D., et al. 2005, A\&A, 439, 997

Quintana, H., Ramirez, A., \& Way, M. J. 1996, AJ, 111, 603

Santos, M. R. 2003, in Extragalactic Globular Cluster Systems, ed. M. KisslerPatig, 348

Sarajedini, A., Geisler, D., Schommer, R., \& Harding, P. 2000, AJ, 120, 2437

Sbordone, L., Bonifacio, P., Marconi, G., Buonanno, R., \& Zaggia, S. 2005, A\&A, 437, 905

Searle, L., \& Zinn, R. 1978, ApJ, 225, 357

Thomas, D., Maraston, C., \& Bender, R. 2003, MNRAS, 339, 897

Thomas, D., Maraston, C., \& Korn, A. 2004, MNRAS, 351, L19

Tolstoy, E., Venn, K. A., Shetrone, M., et al. 2003, AJ, 125, 707

Trager, S. C., Worthey, G., Faber, S. M., Burstein, D., \& Gonzalez, J. J. 1998, ApJS, 116, 1

Whiting, A. B. 1999, AJ, 117, 202

Worthey, G., \& Ottaviani, D. L. 1997, ApJS, 111, 377

Worthey, G., Faber, S. M., Gonzalez, J. J., \& Burstein, D. 1994, ApJS, 94, 687

Zinn, R. 1985, ApJ, 293, 424 
M. D. Mora et al.: Spectroscopy of globular clusters in NGC 45, Online Material $p 1$
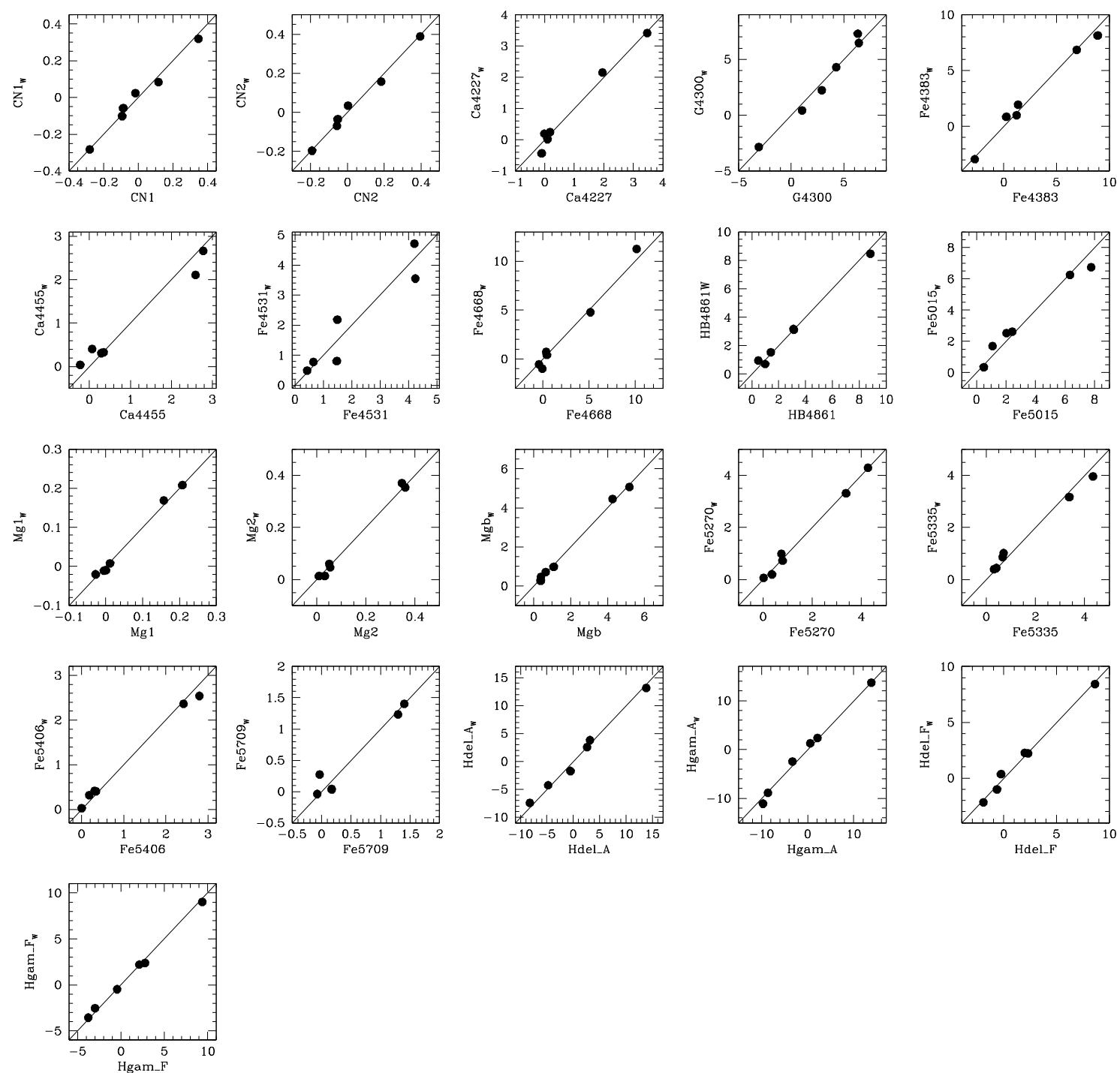

Fig. 6. Comparison of Worthey Lick/IDS indices with the one measured by us. Lines represent the one-to-one comparison. 


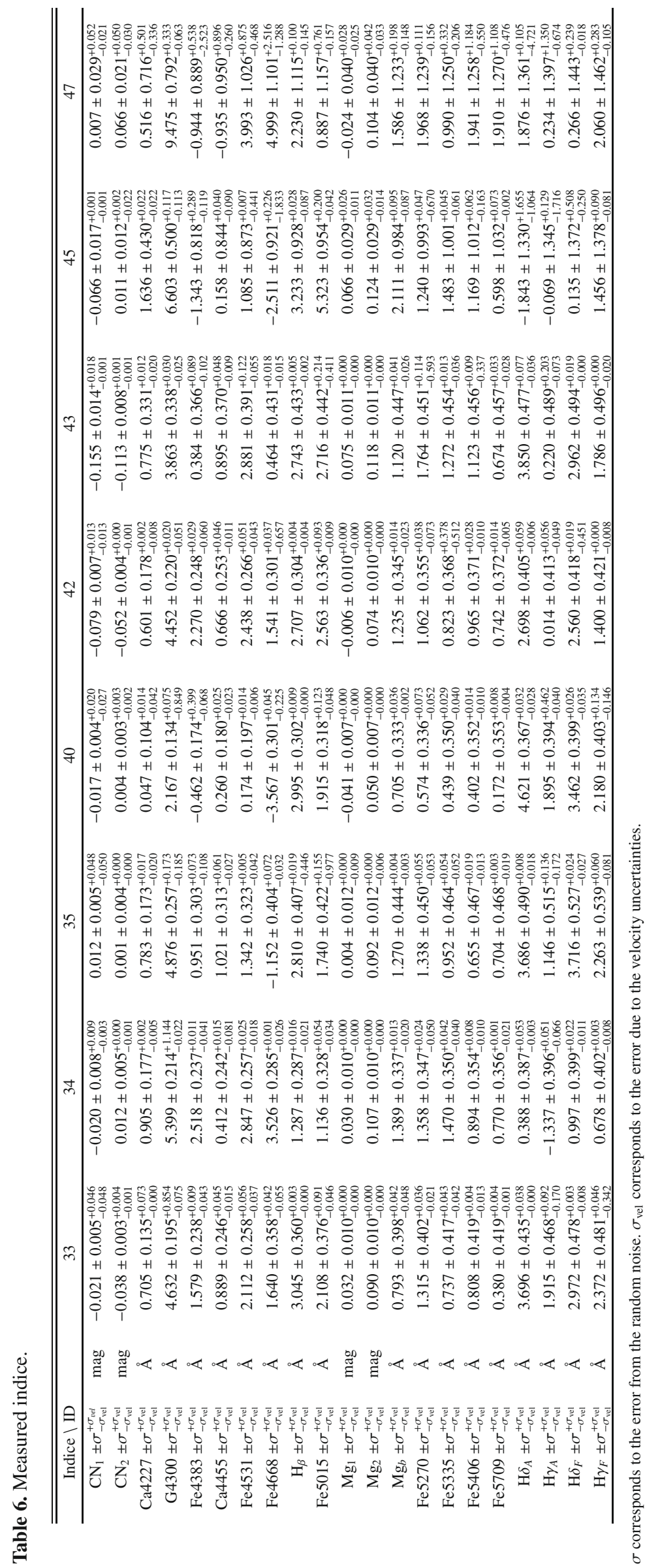

